\title{
Measurement of the Beam-Spin Azimuthal Asymmetry Associated with Deeply-Virtual Compton Scattering
}

A. Airapetian, ${ }^{31}$ N. Akopov, ${ }^{31}$ Z. Akopov ${ }^{31}$ M. Amarian, ${ }^{26,31}$ E.C. Aschenauer, ${ }^{7}$ H. Avakian, ${ }^{11}$ R. Avakian, ${ }^{31}$

A. Avetissian, ${ }^{31}$ E. Avetissian, ${ }^{31}$ P. Bailey,${ }^{15}$ B. Bains, ${ }^{15}$ V. Baturin,${ }^{24}$ C. Baumgarten,${ }^{21}$ M. Beckmann, ${ }^{12}$

S. Belostotski, ${ }^{24}$ S. Bernreuther ${ }^{29}$ N. Bianchi, ${ }^{11}$ H. Böttcher,${ }^{7}$ A. Borissov,${ }^{6,19}$ O. Bouhali, ${ }^{23}$ M. Bouwhuis, ${ }^{15}$

J. Brack,${ }^{5}$ S. Brauksiepe, ${ }^{12}$ W. Brückner, ${ }^{14}$ A. Brüll,${ }^{18}$ I. Brunn,,${ }^{9}$ H.J. Bulten, ${ }^{23,30}$ G.P. Capitani, ${ }^{11}$ P. Chumney, ${ }^{22}$

E. Cisbani,${ }^{26}$ G. Ciullo, ${ }^{10}$ G.R. Court,${ }^{16}$ P.F. Dalpiaz,${ }^{10}$ R. De Leo, ${ }^{3}$ L. De Nardo, ${ }^{1}$ E. De Sanctis,${ }^{11}$

D. De Schepper, ${ }^{2}$ E. Devitsin, ${ }^{20}$ P.K.A. de Witt Huberts, ${ }^{23}$ P. Di Nezza, ${ }^{11}$ M. Düren, ${ }^{9}$ M. Ehrenfried, ${ }^{7}$

G. Elbakian, ${ }^{31}$ F. Ellinghaus, ${ }^{7}$ J. Ely,${ }^{5}$ R. Fabbri, ${ }^{10}$ A. Fantoni, ${ }^{11}$ A. Fechtchenko,${ }^{8}$ L. Felawka, ${ }^{28}$ B.W. Filippone ${ }^{4}$

H. Fischer, ${ }^{12}$ B. Fox,${ }^{5}$ J. Franz,${ }^{12}$ S. Frullani, ${ }^{26}$ Y. Gärber, ${ }^{7,9}$ F. Garibaldi, ${ }^{26}$ E. Garutti ${ }^{23}$ G. Gavrilov,${ }^{24}$

V. Gharibyan, ${ }^{31}$ A. Golendukhin,${ }^{6,21,31}$ G. Graw ${ }^{21}$ O. Grebeniouk, ${ }^{24}$ P.W. Green, ${ }^{1,28}$ L.G. Greeniaus, ${ }^{1,28}$ A. Gute, ${ }^{9}$

W. Haeberli, ${ }^{17}$ K. Hafidi, ${ }^{2}$ M. Hartig, ${ }^{28}$ D. Hasch,${ }^{9,11}$ D. Heesbeen, ${ }^{23}$ F.H. Heinsius, ${ }^{12}$ M. Henoch, ${ }^{9}$

R. Hertenberger ${ }^{21}$ W.H.A. Hesselink,${ }^{23,30}$ G. Hofman,${ }^{5}$ Y. Holler,${ }^{6}$ R.J. Holt,${ }^{15}$ B. Hommez ${ }^{13}$ G. Iarygin, ${ }^{8}$

A. Izotov,${ }^{24}$ H.E. Jackson, ${ }^{2}$ A. Jgoun,${ }^{24}$ P. Jung,${ }^{7}$ R. Kaiser,${ }^{7}$ J. Kanesaka, ${ }^{29}$ E. Kinney, ${ }^{5}$ A. Kisselev,${ }^{2,24}$

P. Kitching, ${ }^{1}$ H. Kobayashi, ${ }^{29}$ N. Koch,${ }^{9}$ K. Königsmann, ${ }^{12}$ H. Kolster, ${ }^{18,23}$ V. Korotkov, ${ }^{7}$ E. Kotik, ${ }^{1}$ V. Kozlov, ${ }^{20}$

B. Krauss, ${ }^{9}$ V.G. Krivokhijine, ${ }^{8}$ G. Kyle, ${ }^{22}$ L. Lagamba,${ }^{3}$ A. Laziev, ${ }^{23,30}$ P. Lenisa, ${ }^{10}$ P. Liebing, ${ }^{7}$ T. Lindemann,${ }^{6}$

W. Lorenzon, ${ }^{19}$ A. Maas,${ }^{7}$ N.C.R. Makins,${ }^{15}$ H. Marukyan, ${ }^{31}$ F. Masoli, ${ }^{10}$ M. McAndrew ${ }^{16}$ K. McIlhany, ${ }^{4,18}$

F. Meissner, ${ }^{9,21}$ F. Menden,${ }^{12}$ N. Meyners,${ }^{6}$ O. Mikloukho,${ }^{24}$ C.A. Miller,${ }^{1,28}$ R. Milner ${ }^{18}$ V. Muccifora,${ }^{11}$

R. Mussa, ${ }^{10}$ A. Nagaitsev, ${ }^{8}$ E. Nappi,${ }^{3}$ Y. Naryshkin,${ }^{24}$ A. Nass,${ }^{9}$ K. Negodaeva, ${ }^{7}$ W.-D. Nowak,${ }^{7}$

K. Oganessyan, ${ }^{6,11}$ T.G. O'Neill, ${ }^{2}$ B.R. Owen, ${ }^{15}$ S.F. Pate,${ }^{22}$ S. Potashov, ${ }^{20}$ D.H. Potterveld, ${ }^{2}$ M. Raithel, ${ }^{9}$

G. Rakness,${ }^{5}$ V. Rappoport,${ }^{24}$ R. Redwine, ${ }^{18}$ D. Reggiani, ${ }^{10}$ A.R. Reolon,,${ }^{11}$ K. Rith,${ }^{9}$ D. Robinson,${ }^{15}$

A. Rostomyan, ${ }^{31}$ M. Ruh, ${ }^{12}$ D. Ryckbosch,${ }^{13}$ Y. Sakemi ${ }^{29}$ I. Sanjiev ${ }^{2,24}$ F. Sato, ${ }^{29}$ I. Savin,${ }^{8}$ C. Scarlett, ${ }^{19}$

A. Schäfer ${ }^{25}$ C. Schill,,${ }^{12}$ F. Schmidt,${ }^{9}$ G. Schnell, ${ }^{22}$ K.P. Schüler, ${ }^{6}$ A. Schwind, ${ }^{7}$ J. Seibert, ${ }^{12}$ B. Seitz,${ }^{1}$

T.-A. Shibata, ${ }^{29}$ V. Shutov,${ }^{8}$ M.C. Simani, ${ }^{23,30}$ A. Simon, ${ }^{12}$ K. Sinram, ${ }^{6}$ E. Steffens, ${ }^{9}$ J.J.M. Steijger, ${ }^{23}$

J. Stewart,,${ }^{2,16,28}$ U. Stösslein, ${ }^{5}$ K. Suetsugu, ${ }^{29}$ S. Taroian, ${ }^{31}$ A. Terkulov ${ }^{20}$ S. Tessarin, ${ }^{10}$ E. Thomas,${ }^{11}$ B. Tipton, ${ }^{4}$

M. Tytgat, ${ }^{13}$ G.M. Urciuoli, ${ }^{26}$ J.F.J. van den Brand, ${ }^{23,30}$ G. van der Steenhoven, ${ }^{23}$ R. van de Vyver, ${ }^{13}$

J.J. van Hunen, ${ }^{23}$ M.C. Vetterli, ${ }^{27,28}$ V. Vikhrov, ${ }^{24}$ M.G. Vincter, ${ }^{1}$ J. Visser, ${ }^{23}$ C. Weiskopf,,${ }^{9}$ J. Wendland ${ }^{27,28}$ J. Wilbert, ${ }^{9}$ T. Wise,${ }^{17}$ S. Yen, ${ }^{28}$ S. Yoneyama, ${ }^{29}$ and H. Zohrabian ${ }^{31}$

(The HERMES Collaboration)

${ }^{1}$ Department of Physics, University of Alberta, Edmonton, Alberta T6G 2J1, Canada

${ }^{2}$ Physics Division, Argonne National Laboratory, Argonne, Illinois 60439-4843, USA

${ }^{3}$ Istituto Nazionale di Fisica Nucleare, Sezione di Bari, 70124 Bari, Italy

${ }^{4}$ W.K. Kellogg Radiation Laboratory, California Institute of Technology, Pasadena, California 91125, USA

${ }^{5}$ Nuclear Physics Laboratory, University of Colorado, Boulder, Colorado 80309-0446, USA

${ }^{6}$ DESY, Deutsches Elektronen Synchrotron, 22603 Hamburg, Germany

${ }^{7}$ DESY Zeuthen, 15738 Zeuthen, Germany

${ }^{8}$ Joint Institute for Nuclear Research, 141980 Dubna, Russia

${ }^{9}$ Physikalisches Institut, Universität Erlangen-Nürnberg, 91058 Erlangen, Germany

${ }^{10}$ Istituto Nazionale di Fisica Nucleare, Sezione di Ferrara and Dipartimento di Fisica, Università di Ferrara, 44100 Ferrara, Italy

${ }^{11}$ Istituto Nazionale di Fisica Nucleare, Laboratori Nazionali di Frascati, 00044 Frascati, Italy

${ }^{12}$ Fakultät für Physik, Universität Freiburg, 79104 Freiburg, Germany

${ }^{13}$ Department of Subatomic and Radiation Physics, University of Gent, 9000 Gent, Belgium

${ }^{14}$ Max-Planck-Institut für Kernphysik, 69029 Heidelberg, Germany

${ }^{15}$ Department of Physics, University of Illinois, Urbana, Illinois 61801, USA

${ }^{16}$ Physics Department, University of Liverpool, Liverpool L69 7ZE, United Kingdom

${ }^{17}$ Department of Physics, University of Wisconsin-Madison, Madison, Wisconsin 53706, USA

${ }^{18}$ Laboratory for Nuclear Science, Massachusetts Institute of Technology, Cambridge, Massachusetts 02139, USA

${ }^{19}$ Randall Laboratory of Physics, University of Michigan, Ann Arbor, Michigan 48109-1120, USA

${ }^{20}$ Lebedev Physical Institute, 117924 Moscow, Russia

${ }^{21}$ Sektion Physik, Universität München, 85748 Garching, Germany

${ }^{22}$ Department of Physics, New Mexico State University, Las Cruces, New Mexico 88003, USA

${ }^{23}$ Nationaal Instituut voor Kernfysica en Hoge-Energiefysica (NIKHEF), 1009 DB Amsterdam, The Netherlands

${ }^{24}$ Petersburg Nuclear Physics Institute, St. Petersburg, Gatchina, 188350 Russia

${ }^{25}$ Institut für Theoretische Physik, Universität Regensburg, 93040 Regensburg, Germany

${ }^{26}$ Istituto Nazionale di Fisica Nucleare, Sezione Roma 1, Gruppo Sanità and Physics Laboratory, Istituto Superiore di Sanità, 
00161 Roma, Italy

${ }^{27}$ Department of Physics, Simon Fraser University, Burnaby, British Columbia V5A 1S6, Canada

${ }^{28}$ TRIUMF, Vancouver, British Columbia V6T 2A3, Canada

${ }^{29}$ Department of Physics, Tokyo Institute of Technology, Tokyo 152, Japan

${ }^{30}$ Department of Physics and Astronomy, Vrije Universiteit, $1081 \mathrm{HV}$ Amsterdam, The Netherlands

${ }^{31}$ Yerevan Physics Institute, 375036, Yerevan, Armenia

(February 4, 2008)

The beam-spin asymmetry in hard electroproduction of photons has been measured for the first time. The data have been accumulated by the HERMES experiment at DESY using the HERA $27.6 \mathrm{GeV}$ longitudinally polarized positron beam and an unpolarized hydrogen gas target. The asymmetry in the azimuthal distribution of the produced photons in the angle $\phi$ relative to the lepton scattering plane was determined with respect to the helicity state of the incoming positron beam. The beam-spin analyzing power in the $\sin \phi$ moment was measured to be $-0.23 \pm 0.04$ (stat) \pm 0.03 (syst) in the missing-mass range below $1.7 \mathrm{GeV}$. The observed asymmetry is attributed to the interference of the Bethe-Heitler and deeply-virtual Compton scattering processes.

PACS numbers: 13.60.Hb, 13.60.Le, 13.60.-r, 24.85.+p 
The internal structure of the nucleon has been extensively studied in deep-inelastic lepton scattering, resulting in such measurements as the momentum distributions of quarks and their helicity dependences. The contribution of the quark spins to the nucleon spin was found to be small. Recently a possibility was identified to study experimentally the total contributions of partons to the nucleon spin, including their orbital angular momenta [1]. This idea is based on the formalism of the so-called skewed parton distributions (SPD) (also referred to as off-forward or generalized parton distributions in the literature [2 5]). In this formalism dynamical correlations between partons with different momenta are taken into account. The SPD framework embodies a wide range of observables, such as electromagnetic form factors, conventional parton distributions and hard exclusive cross sections. In particular, sum rules [5] relate second moments of certain SPDs with the total angular momenta of the quarks and of the gluons in the nucleon.

$A$ reaction that can be cleanly interpreted in terms of SPDs is deeply-virtual Compton scattering (DVCS), i.e. the exclusive leptoproduction of a single multi-GeV photon with the target nucleon remaining intact. Unfortunately, experimental information on DVCS is scant. A central issue is that it is impossible to distinguish between photons originating from DVCS and those from the Bethe-Heitler (BH) process, which can be much more copious. The corresponding diagrams are shown in Fig. 11. However, the interference between the DVCS and $\mathrm{BH}$ processes can be exploited in order to obtain information on DVCS amplitudes. For that purpose the HERMES collaboration has measured the beam-spin asymmetry in hard exclusive electroproduction of photons. The data obtained are presented in this paper.

Using the notation of Ref. [8], the cross section for exclusive leptoproduction of photons can be written as

$$
\frac{d^{4} \sigma}{d \phi d t d Q^{2} d x}=\frac{x y^{2}}{32(2 \pi)^{4} Q^{4}} \frac{\left|\tau_{\mathrm{BH}}+\tau_{\mathrm{DVCS}}\right|^{2}}{\left(1+4 x^{2} m^{2} / Q^{2}\right)^{1 / 2}},
$$

where $x$ represents the Bjorken scaling variable, $y=\nu / E$ the fraction of the incident lepton energy $E$ carried by the virtual photon, $\nu$ its energy and $-Q^{2}$ its four-momentum squared, $m$ the proton mass, and $\tau_{\mathrm{BH}}$ and $\tau_{\mathrm{DVCS}}$ are the $\mathrm{BH}$ and DVCS amplitudes. The cross section shown is differential in $x, Q^{2}, \phi$ and $t$, where the azimuthal angle $\phi$ is the angle between the lepton scattering plane and the plane defined by the virtual and real photons, and $t$ represents the square of the four-momentum transfer to the target.

In Ref. [8] expressions are given for the DVCS $+\mathrm{BH}$ cross sections in leading order $\mathcal{O}(1 / Q)$. (An alternative approach can be found in Ref. [9], for instance.) The leading-order interference term that depends on the helicity of the incident lepton is

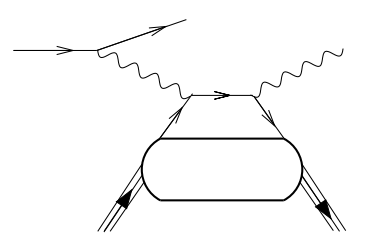

(a)

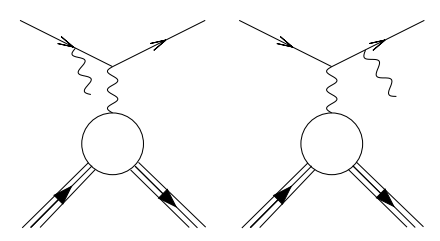

(b)
FIG. 1. (a) Feynman diagram for deeply-virtual Compton scattering, and (b) photon radiation from the incident and scattered lepton in the Bethe-Heitler process.

$$
\begin{array}{r}
\left(\tau_{\mathrm{BH}}^{*} \tau_{\mathrm{DVCS}}+\tau_{\mathrm{DVCS}}^{*} \tau_{\mathrm{BH}}\right)_{p o l}=\frac{4 \sqrt{2} m e^{6}}{t Q x} \cdot \frac{1}{\sqrt{1-x}} \\
\times e_{l} P_{l}\left[-\sin \phi \cdot \sqrt{\frac{1+\epsilon}{\epsilon}} \operatorname{Im} \tilde{M}^{1,1}\right] .
\end{array}
$$

The quantity $\tilde{M}^{1,1}$ is the linear combination of DVCS helicity amplitudes that contributes in the case of a polarized beam and an unpolarized target. The interference is seen to depend on the azimuthal angle $\phi$, the sign of the lepton charge $e_{l}$, and the polarization $P_{l}$ of the incident lepton. The kinematic quantity $\epsilon$ is the polarization parameter of the virtual photon. A determination of the $\sin \phi$-moment of the asymmetry of the interference term shown in Eq. (2) with respect to the beam polarization provides information on the imaginary part of the DVCS amplitude combination $\tilde{M}^{1,1}$, which is related to the SPDs [8]. Not shown in Eq. (2) are other interference terms that are suppressed by $\mathcal{O}(1 / Q)$, but they involve other $\phi$-moments.

The data presented here were recorded during the 1996 and 1997 running periods of the HERMES experiment using the 27.6 GeV HERA longitudinally polarized positron beam at DESY [10]. The beam polarization was continuously measured by Compton back scattering and had an average value of 0.55 with a fractional uncertainty of $3.8 \%$ 11, 12]. The positrons were scattered off a hydrogen gas target [13]. Both unpolarized and spin-averaged polarized-target data have been used in the analysis.

The scattered positrons and coincident photons were detected by the HERMES spectrometer 14 in the polarangle range of 40 to $220 \mathrm{mrad}$. A positron trigger was formed from a coincidence between three scintillator hodoscope planes and a lead-glass calorimeter. The trigger required an energy of more than $3.5 \mathrm{GeV}$ deposited in the calorimeter. Charged particle identification was based on information from four detectors: a threshold Čerenkov counter, a transition radiation detector, a preshower scintillator counter and a lead-glass calorimeter. The particle identification provides an average positron identification efficiency of $99 \%$ with a hadron contamination of less than $1 \%$. Photons are identified by the detection of energy deposition in the calorimeter and preshower counter without an associated charged track. 

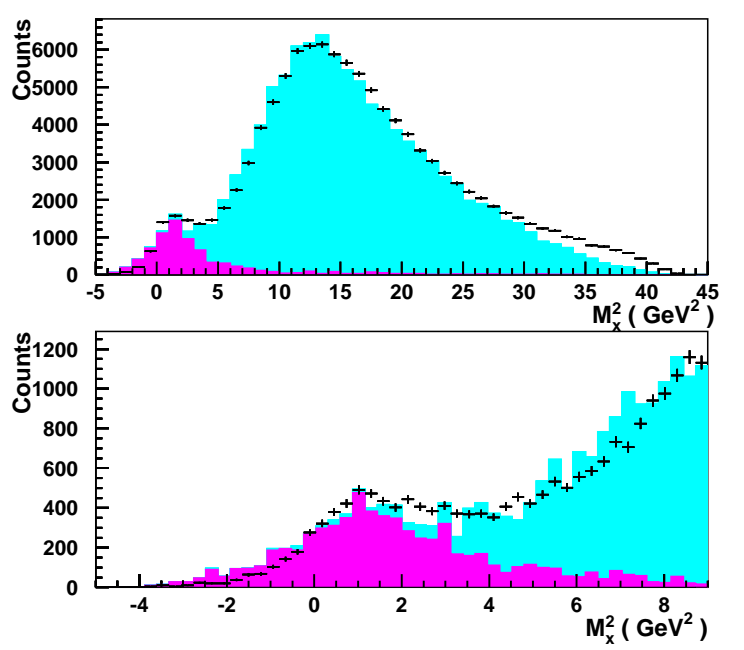

FIG. 2. The measured distribution of photons observed in hard electroproduction versus the missing mass squared $M_{x}{ }^{2}$. In the upper panel the full kinematic range is displayed, while the low $M_{x}{ }^{2}$ domain is shown in the lower panel. The light-gray histogram represents the results of a Monte-Carlo simulation in which fragmentation processes and the Bethe-Heitler process are included, while the dark-shaded histogram represents only the BH contribution. The Monte-Carlo simulation includes the effect of the detector resolution.

Events were selected if they contained only one positron track with momentum larger than $3.5 \mathrm{GeV}$ and only one photon with an energy deposition greater than $0.8 \mathrm{GeV}$ in the calorimeter. The following requirements were imposed on the positron kinematics: $Q^{2}>1 \mathrm{GeV}^{2}$, $W^{2}>4 \mathrm{GeV}^{2}$, and $\nu<24 \mathrm{GeV}$, where $W$ denotes the photon-nucleon invariant mass.

In Fig. 2, the missing mass distribution of the selected events is compared to the results of a Monte-Carlo (MC) simulation in which photons from fragmentation processes in deep-inelastic scattering and from the exclusive $\mathrm{BH}$ process, $e+p \rightarrow e^{\prime}+\gamma+p$, are included. The missing mass is defined as $M_{x}^{2}=\left(q+P_{p}-k\right)^{2}$ with $q, P_{p}$ and $k$ being the four-momenta of the virtual photon, the target nucleon and the produced real photon, respectively. Due to the finite momentum resolution of the spectrometer $M_{x}^{2}$ may be negative, in which case we define $M_{x}=-\sqrt{-M_{x}^{2}}$.

The MC calculation is normalized to the same number of deep-inelastically scattered positrons as were observed inclusively in the experiment (about 5.1 million DIS events), which corresponds to an integrated luminosity of $104 \mathrm{pb}^{-1}$. There is fairly good agreement between the data and the MC results in the relevant kinematic range of the photon spectrum. In the region of low missing mass, the main contribution is due to the $\mathrm{BH}$ process, while the smeared DIS contribution is almost negligible. The smearing of the data to negative $M_{x}^{2}$ values is well reproduced by the Monte-Carlo calculation, which does not include the DVCS process. This result is consistent

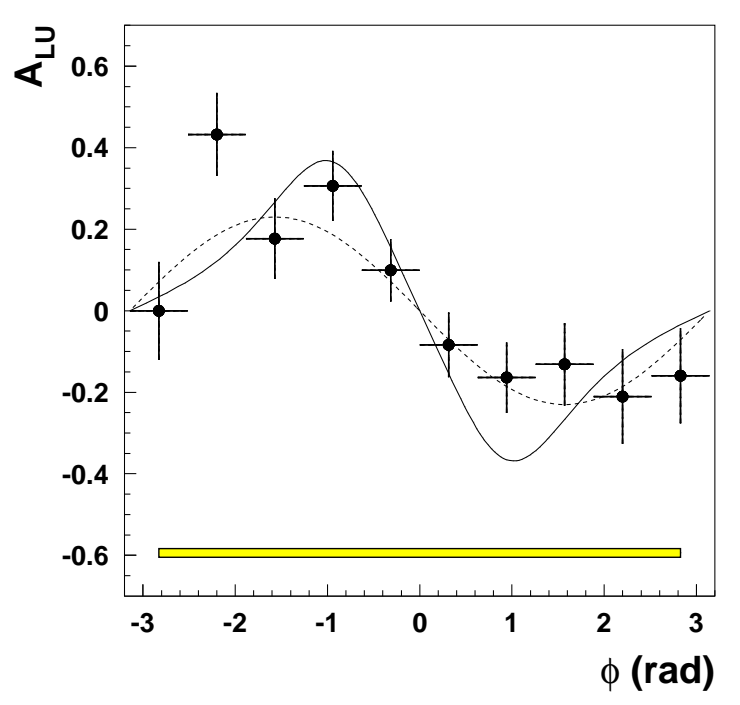

FIG. 3. Beam-spin asymmetry $A_{L U}$ for hard electroproduction of photons as a function of the azimuthal angle $\phi$. The data correspond to the missing mass region between -1.5 and $+1.7 \mathrm{GeV}$. The dashed curve represents a $\sin \phi$ dependence with an amplitude of 0.23 , while the solid curve represents the result of a model calculation taken from Ref. [17]. The horizontal error bars represent the bin width, and the error band below represents the systematic uncertainty.

with the calculations of Ref. 15, where it is shown that the DVCS contribution to the electroproduction of photons is less than $10 \%$ for the present kinematics.

Photon pairs from $\pi^{0}$ decay are removed from the data by requiring the presence of exactly one photon cluster in the (segmented) calorimeter. There could be a remaining $\pi^{0}$ contamination from photon pairs that can not be spatially resolved by the granularity of the calorimeter. It may also happen that one of the $\pi^{0}$ decay photons escapes detection. These contaminations have been estimated in the nominal exclusive region using a MonteCarlo simulation. It was found that $\pi^{0}$ mesons produced in exclusive processes or as fragmentation products in deep-inelastic scattering may contaminate the exclusive part of the photon spectrum by at most $8.5 \%$.

The DVCS-BH interference terms can be extracted from the dependence of the data on the azimuthal angle $\phi$. In order to have an almost full $\phi$-coverage, events were selected with $15<\theta_{\gamma \gamma^{*}}<70 \mathrm{mrad}$, where $\theta_{\gamma \gamma^{*}}$ represents the angle between the directions of the virtual photon and the real photon. A MC-simulation shows that for angles smaller than $15 \mathrm{mrad}$, the granularity of the calorimeter $\left(9 \times 9 \mathrm{~cm}^{2}\right)$ is insufficient to reliably determine the angle $\phi$. For angles larger than $70 \mathrm{mrad}$, the $\phi$-acceptance is restricted. The average $\phi$-resolution in the selected $\theta_{\gamma \gamma^{*}}$ range is about $0.14 \mathrm{rad}$.

In Fig. 3, the azimuthal dependence of the measured beam-spin asymmetry $A_{L U}$ is shown, which is defined as

$$
A_{L U}(\phi)=\frac{1}{\left\langle\left|P_{l}\right|\right\rangle} \cdot \frac{N^{+}(\phi)-N^{-}(\phi)}{\left.N^{+}(\phi)+N^{-}(\phi)\right)}
$$




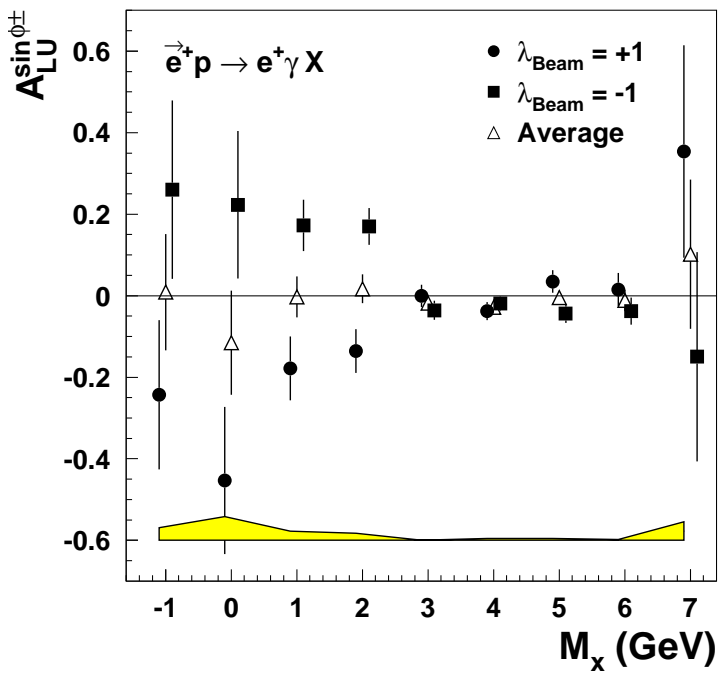

FIG. 4. The $\sin \phi$-moment $A_{L U}^{\sin \phi^{ \pm}}$as a function of the missing mass for positive beam helicity (circles), negative beam helicity (squares) and the averaged helicity (open triangles). A negative value is assigned to $M_{x}$ if $M_{x}^{2}<0$. The error bars are statistical only. The systematic uncertainty is represented by the error band at the bottom of the figure.

where $N^{+}$and $N^{-}$represent the luminosity-normalized yields of events with corresponding beam helicity states, $\left\langle\left|P_{l}\right|\right\rangle$ is the average magnitude of the beam polarization, and the subscripts $L$ and $U$ denote a longitudinally polarized beam and an unpolarized target. The data displayed in Fig. 3 have been selected requiring a missing mass between -1.5 and $+1.7 \mathrm{GeV}$, i.e. $-3 \sigma$ below and $+1 \sigma$ above $M_{x}=m$, and represent 4015 events. An asymmetric $M_{x}$-range was chosen to minimize the influence of the DIS-fragmentation background while optimizing the statistics. Both the proton and the $\Delta(1232)$-resonance are included in the selected $M_{x}$ range. However, the data most likely originate from the exclusive final state with one proton, since the scattering process is dominated by the elastic contribution at kinematics relevant for the $\mathrm{BH}$ process. This conclusion is supported experimentally by figure 2, where the elastic BH Monte Carlo gives a good account of the photon spectrum at low $M_{x}$, and theoretically in Ref. [16]. The comparison of the $A_{L U}$ data in Fig. 3 to a simple $\sin \phi$ curve demonstrates that the data have the $\phi$-dependence expected from Eq. (2). The model calculation of Ref. [17] which is based on the SPD framework and computed at the average kinematics of the present experiment has also been displayed.

In order to be able to compare the $\phi$-dependence of the beam-spin asymmetry for various missing mass bins, the $\sin \phi$-weighted moments have been determined:

$$
A_{L U}^{\sin \phi^{ \pm}}=\frac{2}{N^{ \pm}} \sum_{i=1}^{N^{ \pm}} \frac{\sin \phi_{i}}{\left|P_{l}\right|_{i}},
$$

where the superscript \pm refers to the helicity of the positron beam. In Fig. $⿴$ the extracted values of $A_{L U}^{\sin \phi^{ \pm}}$

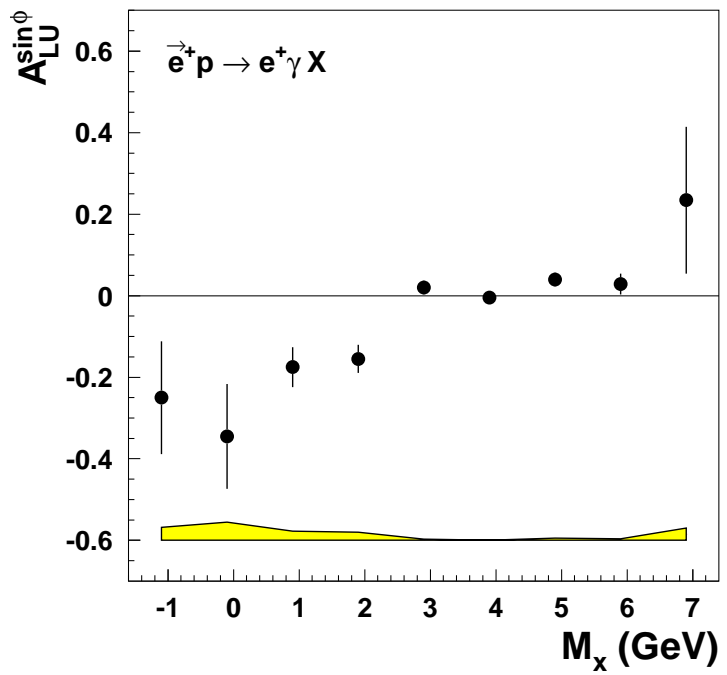

FIG. 5. The beam-spin analyzing power $A_{L U}^{\sin \phi}$ for hard electroproduction of photons on hydrogen as a function of the missing mass. The systematic uncertainty is represented by the error band at the bottom of the figure.

are plotted versus the missing mass $M_{x}$ for the two helicity states $\lambda_{\text {Beam }}$ of the positron beam. The sign of the $\sin \phi$-moment is opposite for the two beam helicities, in agreement with the expectations for the helicity dependence of the relevant DVCS-BH interference term. The beam-spin averaged data are consistent with zero, which is in agreement with the expectations for unpolarized beam and target. The beam-spin averaged data can be used to determine an upper limit of a possible false asymmetry due to instrumental effects which - averaged for $M_{x}$ between -1.5 and $+1.7 \mathrm{GeV}$ - amounts to -0.03 \pm 0.04 .

As the data in Fig. 1 for the two beam-helicity states contain the same physics information, they are combined when evaluating the beam-spin analyzing power $A_{L U}^{\sin \phi}$ :

$$
A_{L U}^{\sin \phi}=\frac{2}{N} \sum_{i=1}^{N} \frac{\sin \phi_{i}}{\left(P_{l}\right)_{i}}
$$

where $N=N^{+}+N^{-}$. In contrast to Eq. (4), the sign of the beam polarization is explicitly taken into account, thus distinguishing the two helicity states. The results are presented in Fig. 5 versus missing mass. All bins in the missing mass region below $M_{x} \approx 2.5 \mathrm{GeV}$ show a similar negative asymmetry, while $A_{L U}^{\sin \phi}$ is consistent with zero for larger $M_{x}$ values. Consequently smeared DIS events at low $M_{x}$ can only marginally dilute the observed asymmetry. As the missing-mass resolution of the HERMES spectrometer for DVCS-like events is $0.77 \mathrm{GeV}$, part of the exclusive data falls below or above $m$. As a result the missing-mass bins left and right of $M_{x}=m$ also show a non-zero value of $A_{L U}^{\mathrm{sin} \phi}$ in Fig. 国.

In order to evaluate the systematic uncertainty on $A_{L U}^{\sin \phi}$ several contributions were considered. The same 
MC simulation described above has been used to estimate the smearing effect on $A_{L U}^{\sin \phi}$, which was found to be less than $5 \%$. The systematic uncertainty associated with smearing and beam polarization is represented by the error bands displayed in Figs. 3, 4, 5. In the exclusive region $\left(-1.5<M_{x}<1.7 \mathrm{GeV}\right)$, two additional contributions to the systematic uncertainty were considered. Possible false asymmetries due to the $\mathrm{BH}$ process are at most $2.6 \%$, while the uncertainty due to the $\pi^{0}$ contamination is estimated to be $12.5 \%$. The total systematic uncertainty at $M_{x} \approx m$ amounts to 0.03 . The quoted instrumental false asymmetry has not been included in this number as it cancels in $A_{L U}^{\sin \phi}$.

By combining the $A_{L U}^{\sin \phi}$ data in the same $M_{x}$ region as was used for Fig. $3\left(-1.5<M_{x}<1.7 \mathrm{GeV}\right)$, an average value of $-0.23 \pm 0.04$ (stat) \pm 0.03 (syst) is obtained. The average values of the kinematic variables corresponding to this measurement are: $\langle x\rangle=0.11,\left\langle Q^{2}\right\rangle$ $=2.6 \mathrm{GeV}^{2}$ and $\langle-t\rangle=0.27 \mathrm{GeV}^{2}$. Since the BH process is dominated by the exclusive final state with one proton 16], and interference can only occur between processes with identical final states, the measured beam-spin analyzing power can be compared to calculations for exclusive processes which are based on the SPD framework. In Ref. 117, e.g., a value of -0.37 is quoted for $A_{L U}^{\sin \phi}$ in a calculation for kinematics close to those of the present experiment. This calculation includes a twist-3 contribution of less than $5 \%$.

In summary, the beam-spin azimuthal asymmetry for hard electroproduction of photons has been measured in the missing mass $\left(M_{x}\right)$ range up to $7 \mathrm{GeV}$. A non-zero asymmetry is observed in the exclusive domain, i.e. for $M_{x} \leq 1.7 \mathrm{GeV}$. The observed $\sin \phi$-moment of the data has the beam-helicity dependence expected from interference between deeply-virtual Compton scattering and the Bethe-Heitler process.

We gratefully acknowledge the DESY management for its support and the staffs at DESY and the collaborating institutions for their significant effort, and our funding agencies for financial support.

[1] X. Ji, Phys. Rev. Lett. 78 (1997) 610.

[2] F-M. Dittes et al., Phys. Lett. B 209 (1988) 325.

[3] D. Müller et al., Fortsch. Phys. 42 (1994) 101.

[4] A.V. Radyushkin, Phys. Lett. B 385 (1996) 333.

[5] X. Ji, Phys. Rev. D 55 (1997) 7114.

[6] B.W. Filippone and X. Ji, Adv. in Nucl. Phys. (2001), in press hep-ph/0101224].

[7] R.L. Jaffe, Nucl. Phys. B 536 (1998) 303.

[8] M. Diehl et al., Phys. Lett. B 411 (1997) 193.

[9] A.V. Belitsky et al., Nucl. Phys. B 593 (2001) 289.

[10] D.P. Barber et al., Phys. Lett. B 343 (1995) 436.
[11] HERMES Collaboration, A. Airapetian et al., Phys. Lett. B 442 (1998) 484.

[12] M. Beckmann et al., Nucl. Instr. Meth. (2001), in press physics/0009047.

[13] J. Stewart. Proc. of the Workshop "Polarized gas targets and polarized beams" eds. R.J. Holt and M.A. Miller, Urbana-Champaign, AIP Conf. Proc. 421 (1997) 69.

[14] HERMES Collaboration, K.Ackerstaff et al., Nucl. Instr. Meth. A 417 (1998) 230.

[15] P.A.M. Guichon and M. Vanderhaeghen, Prog. Nucl. Part. Phys. 41 (1998) 125; M. Vanderhaeghen, P.A.M. Guichon and M. Guidal, Phys. Rev. Lett. 80 (1998) 5064.

[16] L.L. Frankfurt, M.V. Polyakov, and M. Strikman, Contribution to the Workshop "Jefferson Lab Physics and Instrumentation with 6-12 GeV Beams", Newport News, VA, June 15-18, 1998, hep-ph/9808449.

[17] N. Kivel, M. Polyakov and M. Vanderhaeghen, Phys. Rev. D 63 (2001) 114014. 